\title{
Self-truncated ionization injection and consequent monoenergetic electron bunches in laser wakefield acceleration
}

\author{
Ming Zeng, ${ }^{1}$ Min Chen, ${ }^{1,2, *}$ Zheng-Ming Sheng, ${ }^{1,2, \dagger}$ Warren B. Mori, ${ }^{3,4}$ and Jie Zhang ${ }^{1,5}$ \\ ${ }^{1}$ Key Laboratory for Laser Plasmas (Ministry of Education), \\ Department of Physics and Astronomy, Shanghai Jiao Tong University, Shanghai 200240, China \\ ${ }^{2}$ Department of Mathematics, Institute of Natural Sciences, \\ and MOE-LSC, Shanghai Jiao Tong University, Shanghai 20040, China \\ ${ }^{3}$ Department of Electrical Engineering, UCLA, Los Angeles, California 90095, USA \\ ${ }^{4}$ Department of Physics and Astronomy, UCLA, Los Angeles, California 90095, USA \\ ${ }^{5}$ Beijing National Laboratory of Condensed Matter Physics, Institute of Physics, CAS, Beijing 100190, China
}

(Dated: December 25, 2013)

\begin{abstract}
The ionization-induced injection in laser wakefield acceleration has been recently demonstrated to be a promising injection scheme. However, the energy spread controlling in this mechanism remains a challenge because continuous injection in a mixed gas target is usually inevitable. Here we propose that by use of certain initially unmatched laser pulses, the electron injection can be constrained to the very front region of the mixed gas target, typically in a length of a few hundreds micro meters determined by laser-driven bubble deformation. Under some optimized conditions, the injection region is well limited within $200 \mu \mathrm{m}$ and the electron beam with central energy of $383 \mathrm{MeV}$, energy spread of $\Delta E_{\mathrm{FWHM}} / E=3.33 \%$, normalized emittance of $3.12 \mathrm{~mm} \cdot \mathrm{mrad}$ and charge of $14.58 \mathrm{pC}$ can be obtained according to particle-in-cell simulations. Both multi-dimensional simulations and theoretical analysis illustrate the effectiveness of this scheme.
\end{abstract}

PACS numbers: 41.75.Jv, 52.38.Kd, 52.65.Rr

The exploiting of the high acceleration gradient in a laser wakefield accelerator (LWFA) leads to a possible way towards compact and low cost high energy accelerators [1-4]. To compete with the radio-frequency accelerators, the LWFA requires studies pursuing high injection qualities. Injection means placing certain amount of electrons into the accelerating and focusing phase with proper velocities that they can be accelerated continuously. It can be either pre-accelerating some electrons to the phase velocity of the wakefield such as the colliding pulse injection scheme [5-7], or slowing down the wake so that some electrons can catch up with the accelerating phase such as the density ramping or transition injection scheme [8-12]. Recently a new injection scheme called the ionization-induced injection was proposed $[13,14]$. This scheme is attractive due to its simple experimental setup and has been experimentally demonstrated by several groups recently [15-17]. Generally the ionization-induced injection scheme utilizes the higher ionization threshold of the K-shell of a high- $Z$ gas (such as nitrogen, oxygen or argon) mixed with a low-Z gas (usually hydrogen or helium) to control the initial phase of the electrons ionized from the K-shell.

Although the ionization-induced injection has the advantage of relatively easy operation, it has large energy spread because the ionization-induced injection usually occurs continuously until the end of the mixed gas or beam loading effects occur $[18,19]$. Efforts have been devoted to reduce the mixed gas length. In 2011, Liu et al. reduced the mixed gas length to $1 \mathrm{~mm}$ by the two-stage accelerating configuration $[20,21]$. In this experiment the first stage, called injector which filled with mixed gas, is about $1 \mathrm{~mm}$ long. The following stage, called accelerator which filled with pure He, provides longer distance acceleration without injection. By this injector-accelerator scheme, a final absolute energy spread

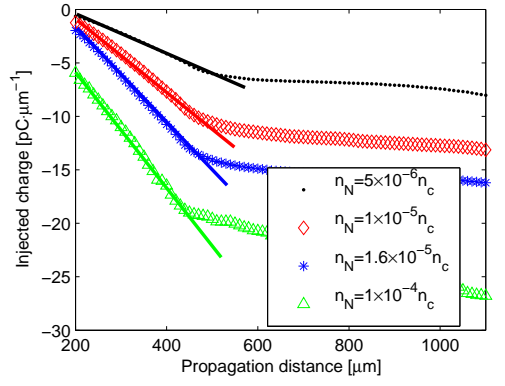

FIG. 1. (Color on line) Injected charge number vs laser propagation distance from 2D PIC simulations with an unmatched laser spot size. Only electrons in the first bucket are considered. The different symbols represent cases with different nitrogen atom density as shown in the legend. The solid lines are linear fittings for data with propagation distance from 200 to $400 \mu \mathrm{m}$. The charge unit is $\mathrm{pC} \cdot \mu \mathrm{m}^{-1}$ due to the $2 \mathrm{D}$ slab geometry.

about $50 \mathrm{MeV}$ is observed. To further reduce the absolute energy spread one needs to reduce the mixed gas length further. However, the up-to-date minimum size of the mixed gas jet is about millimeter scale, which is still longer than satisfactory. Xia et al. demonstrated the quasi-monoenergetic beam generation by controlling the amount of charge from ionization-induced injections by using the laser power close to the ionization threshold of the inner shell of oxygen [22]. In their work, the injected electron energy spread is optimized to about $50 \mathrm{MeV}$ by adjusting the laser peak intensity. The process is both self-focusing and pump-depletion controlled and thus the optimization is not satisfactory. Other efforts such as using separated lase pulses to cut the gas profile are also devoted [23]. Although these experiments can improve the 
final beam quality, usually complicated experimental configurations are required. All optical cutting in ionization based electron injection in LWFA and low energy spread beam production has not been reported.

In this letter, we propose a simple optical method to cut the injection length down to a few hundred micron meters with single stage only, which is much shorter than the mechanical limits obtained so far. This method utilizes the self-focusing process $[24,25]$ of an initially unmatched laser pulse which can automatically truncate the injection process due to bubble deformation before the mixed gas ends. Other than in Ref [22] which use self-focusing to start the ionization-induced injections, the self-focusing of the driver pulse in our work leads to a strong wakefield evolution which later breaks the ionizationinduced injection condition and suppresses the injection process. The resulted injection length is shown to be determined by the period of the laser spot shrinks to the minimum size, which is usually a few hundred microns. This provides a simple way for high quality (low energy spread) ionizationinduced injections.

In our simulations, the gas target is composed of nitrogen and helium mixed gas. The mixed gas is initially uniformly distributed except the $200 \mu \mathrm{m}$ up-ramp from the vacuum to the gas. The simulations are performed in OSIRIS 2.0 framework with the moving window scheme so that the gas target can be half-infinite [26]. The simulation box size is $50 \times 100 \mu \mathrm{m}^{2}$, the cell size is $0.015625 \times 0.25 \mu \mathrm{m}^{2}$ and the time step interval is 0.05 fs. The helium density is kept to be $1.6 \times 10^{-3} n_{\mathrm{c}}$ in all simulations, where $n_{\mathrm{c}}=1.745 \times 10^{21} \mathrm{~cm}^{-3}$ is the critical density for the $800 \mathrm{~nm}$ laser. The nitrogen atom density $\left(n_{\mathrm{N}}\right)$ is relative small and varies from $5 \times 10^{-6} n_{\mathrm{c}}$ to $1 \times 10^{-4} n_{\mathrm{c}}$. Due to the small concentration of nitrogen, the beam loading effects of the injected electrons are negligible, which will be explained in details in the following. The laser pulse is S-polarized in twodimensional (2D) slab geometry simulations with wavelength of $0.8 \mu \mathrm{m}$ and pulse duration of $L_{\mathrm{FWHM}}=33 \mathrm{fs}$. The laser amplitude and focal waist parameters vary either at the so-called matched or unmatched condition for self-guiding [3].

To study the injection process, we first study the charge injection rate along the laser propagation distance in 2D-PIC simulations. The laser beam has a normalized vector potential of $a_{0}=e A / m c^{2}=2.0$ and the waist of $k_{\mathrm{p}} W_{0}=7.594$. Fig. 1 shows the injected beam charges vs laser propagation distance from simulations with different nitrogen concentration. One may notice in all the cases the injected beam charge saturates almost over the same laser propagation distance, which is around $400 \sim 450 \mu \mathrm{m}$. This phenomenon does not agree with the common understanding of the ionization-induced injection process. Usually ionization-induced injection will be continuous if the mixed gas length is long enough, or it will stop due to beam loading effects. According to the equation of maximum affordable number of electrons in the bubble regime $N \simeq 2.5 \times 10^{9} \lambda[\mu \mathrm{m}] / 0.8 \cdot \sqrt{P[\mathrm{TW}] / 100}$ [3], the beam loading should occur when the charge approaches $249 \mathrm{pC}$ (or $36 \mathrm{pC} / \mu \mathrm{m}$ in $2 \mathrm{D}$ slab geometry if we assume the width of the beam is about $7 \mu \mathrm{m}$, which is a common beam width). In our
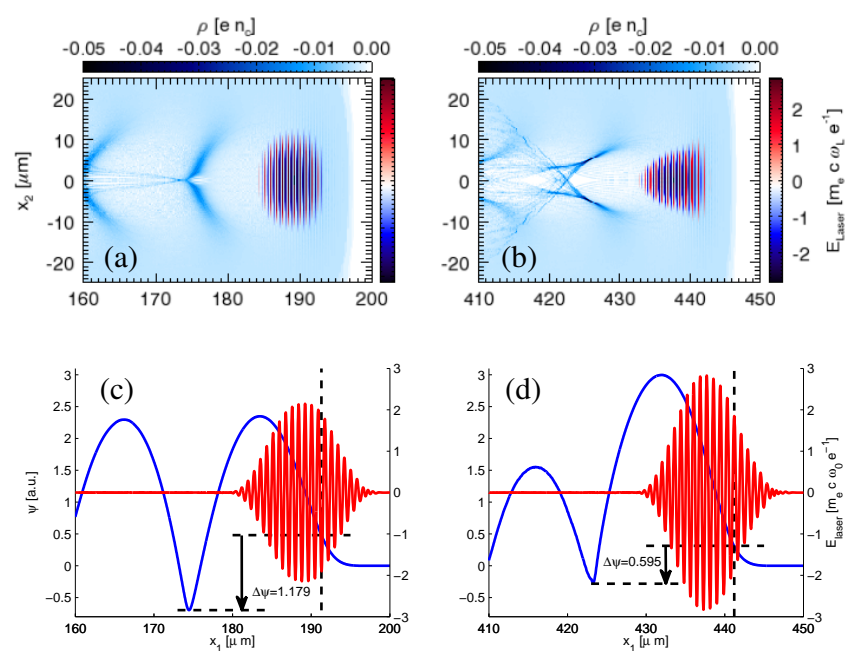

FIG. 2. (Color on line) (a, b) Electron density and laser electric field distribution at the laser propagation distance of 200 and $450 \mu \mathrm{m}$, respectively. (c, d) Laser (red lines) and pseudo-potential (blue lines) line-outs at these two different time steps. Densities are normalized by $n_{\mathrm{c}}=1.745 \times 10^{21} \mathrm{~cm}^{-3}$ and electric fields are normalized by $E_{0}=m_{e} c \omega_{\mathrm{L}} e^{-1}=4 \times 10^{12} \mathrm{~V} / \mathrm{m}$. The dashed lines show the ionization starting point of nitrogen inner shell and the fall to the bottom of the potential well ( $\Delta \psi=1.179$ and 0.595 , respectively).

case, the charge is proportional to the nitrogen concentration. This proves that the injected charges (at least for the low nitrogen concentration cases) are lower than that can trigger the beam loading effect. So the only reason for this kind of injection truncation is due to the shortening of the effective injection length by other mechanisms.

In order to see what induces the injection and cutoff processes, we plot the wake, laser field and the pseudo-potential of the wakefield at two different time steps in Fig. 2. From the static model of plasma based accelerators [17, 19, 27], one knows that the ionization-induced injection occurs when the pseudo-potential difference between the electron ionization position and the end of the wake bucket satisfies $\Delta \psi \geq 1$. Figure 2(a, c) show that at the first selected time step the laser pulse still holds its gaussian shape and $\Delta \psi$ of the excited wake is large enough for the ionization-induced injection to occur. However at the second selected position $(450 \mu \mathrm{m})$ the laser is strongly deformed and self-focused, and the profile is transformed to a bell-like shape (front steepened), meanwhile the laser field amplitude is about $45 \%$ higher than initial. This larger amplitude laser pulse excites a wake with a larger amplitude (see Fig. 2(d)). The minimum potential increases which makes $\Delta \psi$ smaller and the ionization-induced injection condition $(\Delta \psi \geq 1)$ is no longer satisfied. This is the reason of the injection truncation. Since the nitrogen concentration in all of our simulations is low, the laser pulse evolution is mainly affected or determined by the background plasmas. The pulse evolution in all of the simulations are almost the same. So the truncation positions of the ionization-induced injections are almost the same regardless of the change of $n_{\mathrm{N}}$. 

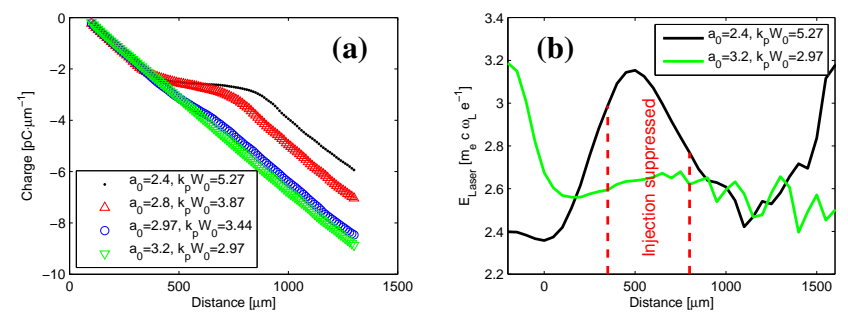

FIG. 3. (Color on line) (a) Injected charge number vs. laser propagation distance from 2D PIC simulations with different normalized laser vector potential $a_{0}$ and normalized waist $k_{\mathrm{p}} W_{0}$. The laser energies are kept the same in 2D slab geometry by keeping $a_{0}^{2} k_{\mathrm{p}} W_{0}$ a constant. (b) Maximum axial laser field evolution of two cases: black line for $a_{0}=2.4, k_{\mathrm{p}} W_{0}=5.27$ and green line for $a_{0}=3.2$, $k_{\mathrm{p}} W_{0}=2.97$. The red dashed lines indicate the region where the injection is suppressed for the black line case.

The above analysis shows that it is the self-evolution of the pulse terminates the ionization-induced injection process. As we know the pulse evolution in the plasma depends on its initial power and focus state. A matched laser pulse can have a very stable transverse profile during its propagation. To check our analysis we make a serials of simulations which keep the pulse power in 2D geometry but varying the pulse's initial transverse focusing size, which affects the pulse's selfevolution.

The matched spot size in relativistic condition (estimated with large $a_{0}$ ), described in Ref. [3], is $k_{\mathrm{p}} W_{0}=2 \sqrt{a_{0}}$, where $k_{\mathrm{p}}$ is the wave number of a plasma wave. Earlier 3D simulations show a laser beam satisfying this matching condition can keep its shape until pump depletion develops. In the following, we compare the ionization induced injection rate between different laser parameters, i. e. the normalized vector potential $a_{0}$ and waist $k_{\mathrm{p}} W_{0}$. The ratio $n_{\mathrm{N}} / n_{\mathrm{H}_{\mathrm{e}}}$ is fixed to be $0.1 \%$ so that the beam loading effect is negligible. In order to keep the laser power in 2D slab geometry, we keep $a_{0}^{2} W_{0}$ to be a constant in different simulation cases, which could be relevant for an asymmetric spot size in 3D.

Figure 3(a) shows the ionization-induced injection rates with different laser parameters. The case $a_{0}=2.4, k_{\mathrm{p}} W_{0}=$ 5.27 shows a clear injection suppression start from about $400 \mu \mathrm{m}$. The power to self-focusing critical power ratio is $P / P_{c}=\alpha\left(k_{\mathrm{p}} W_{0} a_{0}\right)^{2} / 32=7.07$ in this case $(\alpha=\sqrt{2}$ for $2 \mathrm{D}$ slab geometry) [28]. The case $a_{0}=2.97, k_{\mathrm{p}} W_{0}=3.44$ is the best matched case from the theory, but the case $a_{0}=3.2$, $k_{\mathrm{p}} W_{0}=2.96$ shows a better linear injection behavior. This discrepancy is because the above matching condition is an estimation with large $a_{0}$. Figure 3(b) shows the evolution of on-axis laser electric field. As one can see, in the most unmatched case with $a_{0}=2.4$ the ionization-induced injection happens periodically. This is due to the pulse's periodically focusing and defocusing and the consequent bubble evolution. The two red dashed vertical lines labels the region where ionization-induced injection is suppressed. In this simulation two bunches of ionization-induce electron injections can be
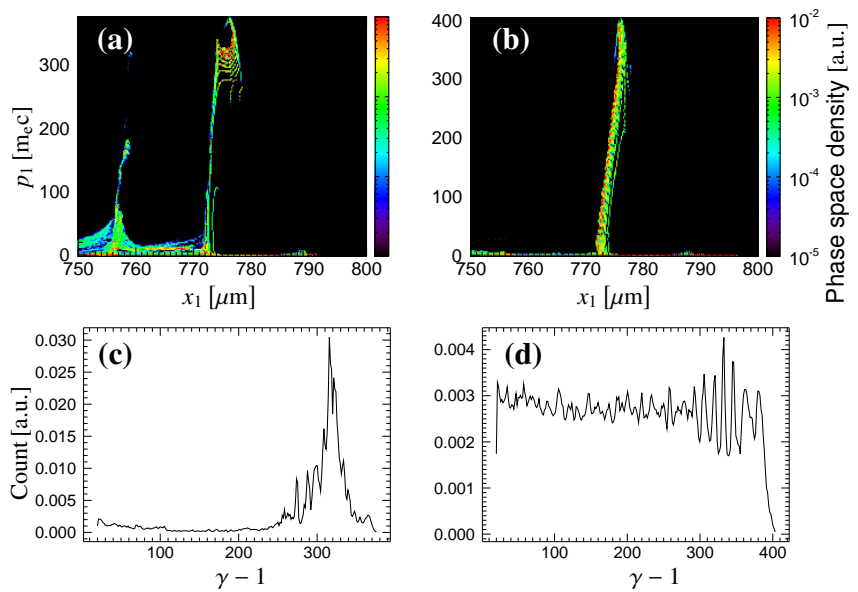

FIG. 4. (Color on line) The phase space $(a, b)$ and the energy spectra (c, d) of the injected electrons for the cases (a, c) $a_{0}=2.4, k_{\mathrm{p}} W_{0}=$ 5.27 and (b, d) $a_{0}=3.2, k_{\mathrm{p}} W_{0}=2.96$, i.e. the black dots and the green triangle in Fig. 3 (a), respectively. The beam qualities are (a, c) $7.1 \mathrm{pC}$ in charge, $1.05 \mathrm{~mm} \cdot \mathrm{mrad}$ in emittance and $7.9 \%$ energy spread in FWHM with central energy of $160 \mathrm{MeV}$, (b, d) 15.9 pC in charge, $1.1 \mathrm{~mm} \cdot \mathrm{mrad}$ in emittance and $100 \%$ energy spread with maximum energy of $200 \mathrm{MeV}$.

seen in the same wake bucket. The final spectrum shows one mono-energetic peak with a low energy background, which has been observed in serval former experiments. However, in most of the reports, this phenomenon is explained by regarding the electrons to be in different buckets.

We compare the phase space and energy spectrum for these two cases in Fig. 4. With the unmatched laser pulse, the injected charge is reduced by a half but the energy spread is greatly improved, and mono-energetic electron beam due to ionization injection can be seen (Fig. 4(a, c)). Simulations prove that slightly increasing the $n_{\mathrm{N}}$ can recover the final injected charge but keep the low energy spread in unmatched cases. In the range of mixing ratio $<1 \%$, the injected charge increases almost linearly with $n_{\mathrm{N}}$. In the matched case, due to the continuous injection, accelerated electron distribution in phase space appears continuous. This results in an almost flat spectrum in the final energy distribution (see Fig. 4(b, d)).

By estimating the self-focusing length, one can optimize the injection for better beam quality. To do this, we consider the equation for laser profile evolution with $d^{2} R / d z^{2}=$ $Z_{\mathrm{R}}^{-2} R^{-3}\left(1-\alpha a^{2} k_{\mathrm{p}}^{2} W^{2} / 32\right)$, where $R=W / W_{0}$ is the normalized spot size and $Z_{\mathrm{R}}=k W_{0}^{2} / 2$ is the vacuum Rayleigh length $[28,29]$. Although it only applies in the weak nonlinear limit, this equation still leads the way towards the optimization in our weak blowout regime as discussed in the follows. For a 3D case, one can obtain the analytic solution $R^{2}=1+\left(1-P / P_{\mathrm{c}}\right) z^{2} / Z_{\mathrm{R}}^{2}$ with the initial condition $\dot{R}=0$ and $R=1$ at $z=0$, which indicates that the laser spot size shrinks to 0 at

$$
z=Z_{\mathrm{R}}\left(P / P_{\mathrm{c}}-1\right)^{-1 / 2},
$$

where $P / P_{\mathrm{c}}=\left(k_{\mathrm{p}} W_{0} a_{0}\right)^{2} / 32$. For the $2 \mathrm{D}$ slab geometry which 

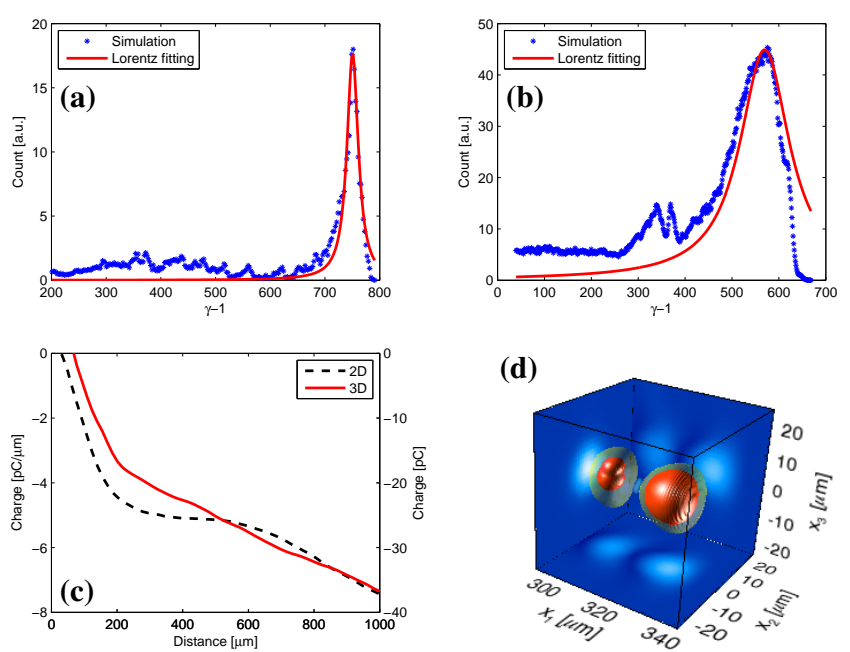

FIG. 5. (Color on line) The optimized 2D simulation and its comparison with the $3 \mathrm{D}$ simulation. (a) The optimized $2 \mathrm{D}$ simulation result with laser parameters $a_{0}=2.9$ and $k_{\mathrm{p}} W_{0}=5.228$. (b) The 3D simulation beam energy spectrum with the same parameters as in the optimized 2D case. The spectrum is taken at propagation distance of $760 \mu \mathrm{m}$. (c) The injected beam charge vs. propagation distance for the 2D simulation shown in (a) and 3D simulation shown in (b). When calculating the propagation distance, the density transition between vacuum and the gas is excluded. The charge zero point is not the distance zero point because it takes a while for the electrons to reach the injection criteria. (d) The pseudo-potential $\psi$ isosurface and its projections for the $3 \mathrm{D}$ wake, which evolves to a nut-like shape at propagation distance of a few hundred microns. The 3D simulation has the same simulation box size as $2 \mathrm{D}$ simulations, but the displayed region is reduced.
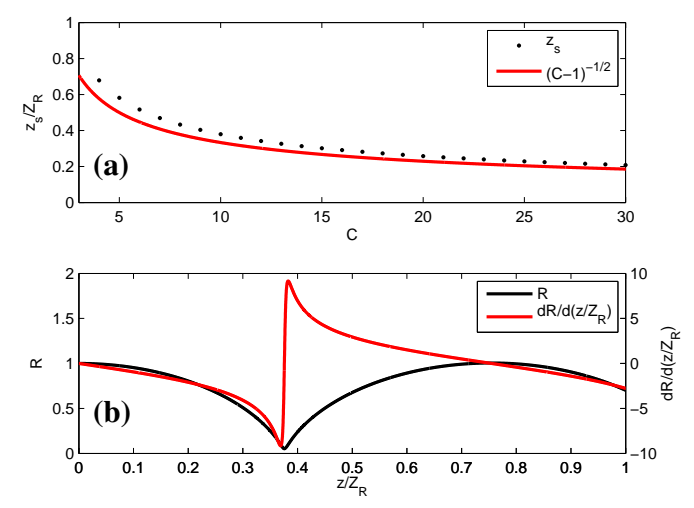

FIG. 6. (Color on line) (a) $z_{\mathrm{s}}$ vs. $C$ and its estimation $(C-1)^{-1 / 2}$ Numerically result of Eq. 2 with the parameter $C=10$.

is mostly used in simulations, the laser profile evolution function becomes

$$
\frac{\mathrm{d}^{2} R}{\mathrm{~d}\left(z / Z_{\mathrm{R}}\right)^{2}}=\frac{1-C \cdot R}{R^{3}},
$$

where $C=\frac{\sqrt{2}}{32} a_{0}^{2} k_{\mathrm{p}}^{2} W_{0}^{2}$ which is a constant determined by the initial laser power. After some integral techniques, the distance $z_{\mathrm{s}}$ at which $R$ shrinks to its minimal $R_{\min }=1 /(2 C-1)$ can be found as

$$
z_{\mathrm{s}} / Z_{\mathrm{R}}=\int_{-\frac{\pi}{2}}^{\frac{\pi}{2}} \frac{\mathrm{d} \theta}{[(C-1) \sin \theta+C]^{2}} .
$$

By numerical integral, one may plot $z_{\mathrm{s}}$ vs. $C$ as shown in Fig. 6 (a). Also, we found from the plot that the 2D counterpart of the $3 \mathrm{D}$ solution Eq. 1 can give a good estimation $z_{\mathrm{s}} \gtrsim Z_{\mathrm{R}}(C-1)^{-1 / 2} \equiv z_{\text {cut }}$ (the same laser parameter leads to a factor of $\sqrt{2}$ difference on $C$ between $2 \mathrm{D}$ and 3D).

We can estimate the injection length to be $z_{\text {cut }}$. The former laser parameters $\left(a_{0}=2\right.$ and $\left.k_{\mathrm{p}} W_{0}=7.594\right)$ gives $z_{\text {cut }}=373 \mu \mathrm{m}$, which is consistent with previous simulation (Fig. 1). Next, we keep the laser power to be the same $\left(P=39 \mathrm{TW}\right.$, thus $\left.a_{0} k_{\mathrm{p}} W_{0}=15.2\right)$ and change the initial $a_{0}$. From a series of simulations we found that the ionizationinduced injections can be sufficiently reduced if $a_{0}>2.9$. Thus if we choose the condition $a_{0}=2.9$, the minimum injection length is obtained to be $178 \mu \mathrm{m}$. The PIC simulations also confirm this estimation (the injection length is reduced to $187 \mu \mathrm{m}$ with this set of parameters). At this condition, the ionization injection leads to the generation of an electron beam with $14.58 \mathrm{pC}$ in charge, $383 \mathrm{MeV}$ in the central energy with energy spread of $\Delta E_{\mathrm{FWHM}} / E=3.33 \%$ (shown in Fig. 5 (a)), and normalized emittance of $3.12 \mathrm{~mm} \cdot \mathrm{mrad}$ at the propagation distance $1.4 \mathrm{~mm}$. In this optimized case, the initial laser parameters are $a_{0}=2.9$ and $W_{0}=11.69 \mu \mathrm{m}$, and the gas is $n_{\mathrm{H}_{\mathrm{e}}}=2.8 \times 10^{18} \mathrm{~cm}^{-3}$ and $n_{\mathrm{N}}=8.5 \times 10^{15} \mathrm{~cm}^{-3}$ mixed. Compared with a very recent published work on two-pulse ionization injection [30], our work has the advantages on simpler configuration using one pulse only and larger charge with similar beam emittance.

In a real case, the pulse evolution is actually a threedimensional (3D) process, therefore 3D simulation is necessary to check if self-truncated ionization injection really exists. The results from the 3D simulation with the same laser parameters as in the 2D optimized case are shown in Fig. 5 (bd). One may notice that in 3D the beam energy spread is larger than in $2 \mathrm{D}$, and the ionization-induced injection is slowed down at $200 \mu \mathrm{m}$ instead of totally suppressed (Fig. 5 (c)). This is because the $3 \mathrm{D}$ symmetric laser pulse has different evolution compared with that in 2D-slab geometry. Nevertheless, the quasi-monoenergetic character still confirms the validity of this injection truncation phenomenon. More simulations towards 3D optimization is our future goal.

In summary we have demonstrated a simple method to shorten the effective injection length in the ionization based scheme. The self-evolution of an initially unmatched laser pulse can break the ionization-induced injection condition and make the injection length much shorter than that for matched laser beam, which makes the final accelerated electron beam mono-energetic. Different from the former researches of the self-injection due to the bubble evolution [31, 32], the injection here is ionization-induced. It deserves to point out that since the shortening of the injection is based on pulse evolution, long distance acceleration for achieving high energy acceleration requires matched spot size at further stages. Nev- 
ertheless, our study is helpful for the initial injection process, which is vital for final beam qualities.

The authors would like to acknowledge the OSIRIS Consortium, consisting of UCLA and IST (Lisbon, Portugal) for the use of OSIRIS and the visXD framework. MZ appreciates the useful discussions with Frank Tsung, Weiming An, Asher Davidson, Feiyu Li and Yue Liu. This work is supported by the National Basic Research Program of China (Grant No. 2013CBA01504) and the National Science Foundation of China (Grants No. 11121504, 11129503, 11075105 and 11205101). Simulations were performed on the Hoffman 2 at UCLA, Hopper at NERSC, and Supercomputer $\Pi$ at Shanghai Jiao Tong University.

minchen@sjtu.edu.cn

zmsheng@sjtu.edu.cn

[1] T. Tajima and J. M. Dawson, Phys. Rev. Lett. 43, 267 (1979).

[2] A. Pukhov and J. Meyer-ter Vehn, Appl. Phys. B 74, 355 (2002).

[3] W. Lu, M. Tzoufras, C. Joshi, F. S. Tsung, W. B. Mori, J. Vieira, R. A. Fonseca, and L. O. Silva, Phys. Rev. ST Accel. Beams 10, 061301 (2007).

[4] K. Nakajima, H. Lu, X. Zhao, B. Shen, R. Li, and Z. Xu, Chin. Opt. Lett. 11, 013501 (2013).

[5] E. Esarey, R. F. Hubbard, W. P. Leemans, A. Ting, and P. Sprangle, Phys. Rev. Lett. 79, 2682 (1997).

[6] J. Faure, C. Rechatin, A. Norlin, A. Lifschitz, Y. Glinec, and V. Malka, Nature 444, 737 (2006).

[7] C. Rechatin, J. Faure, A. Ben-Ismail, J. Lim, R. Fitour, A. Specka, H. Videau, A. Tafzi, F. Burgy, and V. Malka, Phys. Rev. Lett. 102, 164801 (2009).

[8] C. G. R. Geddes, K. Nakamura, G. R. Plateau, C. Toth, E. Cormier-Michel, E. Esarey, C. B. Schroeder, J. R. Cary, and W. P. Leemans, Phys. Rev. Lett. 100, 215004 (2008).

[9] A. Gonsalves, K. Nakamura, C. Lin, D. Panasenko, S. Shiraishi, T. Sokollik, C. Benedetti, C. Schroeder, C. Geddes, J. Van Tilborg, et al., Nature Phys. 7, 862 (2011).

[10] S. Bulanov, N. Naumova, F. Pegoraro, and J. Sakai, Phys. Rev. E 58, R5257 (1998).

[11] M. Zeng, N. A. M. Hafz, K. Nakajima, L.-M. Chen, W. Lu, W. B. Mori, Z.-M. Sheng, and J. Zhang, J. Plasma Phys. 78, 363 (2012).

[12] F. Y. Li, Z. M. Sheng, Y. Liu, J. Meyer-ter Vehn, W. B. Mori, W. Lu, and J. Zhang, Phys. Rev. Lett. 110, 135002 (2013).

[13] M. Chen, Z.-M. Sheng, Y.-Y. Ma, and J. Zhang, J. Appl. Phys. 99, 056109 (2006).

[14] E. Oz, S. Deng, T. Katsouleas, P. Muggli, C. D. Barnes, I. Blumenfeld, F. J. Decker, P. Emma, M. J. Hogan, R. Ischebeck,
R. H. Iverson, N. Kirby, P. Krejcik, C. O’Connell, R. H. Siemann, D. Walz, D. Auerbach, C. E. Clayton, C. Huang, D. K. Johnson, C. Joshi, W. Lu, K. A. Marsh, W. B. Mori, and M. Zhou, Phys. Rev. Lett. 98, 084801 (2007).

[15] C. E. Clayton, J. E. Ralph, F. Albert, R. A. Fonseca, S. H. Glenzer, C. Joshi, W. Lu, K. A. Marsh, S. F. Martins, W. B. Mori, A. Pak, F. S. Tsung, B. B. Pollock, J. S. Ross, L. O. Silva, and D. H. Froula, Phys. Rev. Lett. 105, 105003 (2010).

[16] C. McGuffey, A. G. R. Thomas, W. Schumaker, T. Matsuoka, V. Chvykov, F. J. Dollar, G. Kalintchenko, V. Yanovsky, A. Maksimchuk, K. Krushelnick, V. Y. Bychenkov, I. V. Glazyrin, and A. V. Karpeev, Phys. Rev. Lett. 104, 025004 (2010).

[17] A. Pak, K. A. Marsh, S. F. Martins, W. Lu, W. B. Mori, and C. Joshi, Phys. Rev. Lett. 104, 025003 (2010).

[18] M. Tzoufras, W. Lu, F. S. Tsung, C. Huang, W. B. Mori, T. Katsouleas, J. Vieira, R. A. Fonseca, and L. O. Silva, Phys. Rev. Lett. 101, 145002 (2008).

[19] M. Chen, E. Esarey, C. B. Schroeder, C. G. R. Geddes, and W. P. Leemans, Phys. Plasmas 19, 033101 (2012).

[20] J. S. Liu, C. Q. Xia, W. T. Wang, H. Y. Lu, C. Wang, A. H. Deng, W. T. Li, H. Zhang, X. Y. Liang, Y. X. Leng, X. M. Lu, C. Wang, J. Z. Wang, K. Nakajima, R. X. Li, and Z. Z. Xu, Phys. Rev. Lett. 107, 035001 (2011).

[21] B. B. Pollock, C. E. Clayton, J. E. Ralph, F. Albert, A. Davidson, L. Divol, C. Filip, S. H. Glenzer, K. Herpoldt, W. Lu, K. A. Marsh, J. Meinecke, W. B. Mori, A. Pak, T. C. Rensink, J. S. Ross, J. Shaw, G. R. Tynan, C. Joshi, and D. H. Froula, Phys. Rev. Lett. 107, 045001 (2011).

[22] C. Xia, J. Liu, W. Wang, H. Lu, W. Cheng, A. Deng, W. Li, H. Zhang, X. Liang, Y. Leng, X. Lu, C. Wang, J. Wang, K. Nakajima, R. Li, and Z. Xu, Phys. Plasmas 18, 113101 (2011).

[23] C.-T. Hsieh, C.-M. Huang, C.-L. Chang, Y.-C. Ho, Y.-S. Chen, J.-Y. Lin, J. Wang, and S.-Y. Chen, Phys. Rev. Lett. 96, 095001 (2006).

[24] G.-Z. Sun, E. Ott, Y. C. Lee, and P. Guzdar, Phys. Fluids 30, 526 (1987).

[25] W. Mori, IEEE J. Quantum Electron. 33, 1942 (1997).

[26] R. A. Fonseca et al., LECTURE NOTES IN COMPUTER SCIENCE, Vol. 2331 (2002) pp. 342-351.

[27] W. Lu, C. Huang, M. Zhou, W. B. Mori, and T. Katsouleas, Phys. Rev. Lett. 96, 165002 (2006).

[28] K.-C. Tzeng and W. B. Mori, Phys. Rev. Lett. 81, 104 (1998).

[29] E. Esarey, C. B. Schroeder, and W. P. Leemans, Rev. Mod. Phys. 81, 1229 (2009).

[30] N. Bourgeois, J. Cowley, and S. M. Hooker, Phys. Rev. Lett. 111, 155004 (2013).

[31] S. Y. Kalmykov, S. A. Yi, V. N. Khudik, and G. Shvets, Phys. Rev. Lett. 103, 135004 (2009).

[32] S. Corde, C. Thaury, A. Lifschitz, G. Lambert, K. T. Phuoc, X. Davoine, R. Lehe, D. Douillet, A. Rousse, and V. Malka, Nat. Commun. 4, 1501 (2013). 